\title{
New Oscillation Criteria for the Second Order Nonlinear Differential Equations with Damping
}

\author{
Quanxin Zhang, Xia Song, Shouhua Liu
}

Department of Mathematics, Binzhou University, Shandong, China

Email: 3314744@163.com, songxia119@163.com, 13169207@163.com

Received 28 May 2016; accepted 27 June 2016; published 30 June 2016

Copyright (C) 2016 by authors and Scientific Research Publishing Inc.

This work is licensed under the Creative Commons Attribution International License (CC BY). http://creativecommons.org/licenses/by/4.0/

(c) (i) Open Access

\begin{abstract}
In this paper, we are concerned with a class of second-order nonlinear differential equations with damping term. By using the generalized Riccati technique and the integral averaging technique of Philos-type, two new oscillation criteria are obtained for every solution of the equations to be oscillatory, which extend and improve some known results in the literature recently.
\end{abstract}

Keywords

Oscillation Criterion, Differential Equations with Damping, Integral Averaging Method

\section{Introduction}

Zhang and Yan discussed respectively the solutions' oscillation of the second order nonlinear differential equation with damping in [1]-[3]

$$
\left(a(t) \psi(x(t)) x^{\prime}(t)\right)^{\prime}+p(t) x^{\prime}(t)+q(t) f(x(t))=0,
$$

and obtained some useful results. On this basis, the paper continues this discussion of Equation (1). For Equation (1), assume that

$\left(A_{1}\right) \quad a:\left[t_{0},+\infty\right) \rightarrow \mathrm{R}(\mathrm{R}=(-\infty,+\infty))$ is continuously differentiable;

$\left(A_{2}\right) \quad p, q:\left[t_{0},+\infty\right) \rightarrow \mathrm{R}$ are continuous functions, and for arbitrarily large $t, p(t), q(t)$ can change sign;

$\left(A_{3}\right) \psi, f: \mathrm{R} \rightarrow \mathrm{R}$ is continuously differentiable and when $u \neq 0, \psi(u)>0, u f(u)>0, f^{\prime}(u)>0$.

In this paper, we assume that each solution $x(t)$ of Equation (1) can be extended to $\left[t_{0},+\infty\right)$. A solution is 
said to be regular if there exists $t$ on arbitrary interval $[T,+\infty)$, such that $x(t) \neq 0$. A regular solution is said to be oscillatory, if it has arbitrarily large zeros; otherwise it is said to be nonoscillatory. Equation (1) is called oscillatory if all its regular solutions are oscillatory.

Many exceptions of Equation (1) have emerged in the literature, for example, the paper [4] discussed the oscillation of the second order linear differential equation with damping

$$
\left(a(t) x^{\prime}(t)\right)^{\prime}+p(t) x^{\prime}(t)+q(t) x(t)=0,
$$

and the associated equations have been studied by many authors with a number of important results of oscillation. We recommend References [5]-[7] and their introductions. The purpose of this paper is to establish the Philos-type oscillation criteria of Equation (1) in general conditions. By using the generalized Riccati transformation and integral averaging technique of Philos-type [8], we obtain three new oscillatory criteria for Equation (1). Our results generalize, improve and unify the above results in above references and the method of proof is also relatively simpler than their's. The functions inequalities in this article are established for all sufficiently large $t$ if there is no particular explanation.

\section{Main Results}

Using Philos-type integral average conditions, the new oscillatory results of Equation (1) is given as below. Function classes $P$ is introduced, we define that

$$
\mathrm{D}_{0}=\left\{(t, s): t>s \geq t_{0}\right\}, \mathrm{D}=\left\{(t, s): t \geq s \geq t_{0}\right\} .
$$

$H \in \mathrm{C}(D, R)$ is called function belong to the class $\mathrm{P}$, if there is $h \in \mathrm{C}\left(D_{0}, R\right)$ satisfying

1) $H(t, t)=0, t \geq t_{0} ; H(t, s)>0,(t, s) \in \mathrm{D}_{0}$;

2) $H$ exists non-positive and continuously partial derivatives for the second variable in $\mathrm{D}_{0}$, and satisfies the equation

$$
-\frac{\partial H(t, s)}{\partial s}=h(t, s) \sqrt{H(t, s)}, \quad(t, s) \in D_{0} .
$$

Theorem 1. Assume that $\left(A_{1}\right)-\left(A_{3}\right)$ hold, and $0<c_{1} \leq \psi(x) \leq c_{2}, f^{\prime}(x) \geq k>0, x \neq 0$. The function $H(t, s)$ belongs to the class of functions $P$ and (4) holds. If there is an continuously differentiable function $\rho(t):\left[t_{0},+\infty\right) \rightarrow(0,+\infty)$ making

$$
\begin{aligned}
& \limsup _{t \rightarrow+\infty} \frac{1}{H\left(t, t_{0}\right)} \int_{t_{0}}^{t}\left\{H(t, s) \rho(s)\left[q(s)+\frac{\left(c_{1}-c_{2}\right) p^{2}(s)}{4 k c_{1} c_{2} a(s)}\right]\right. \\
& \left.-\frac{1}{4}\left[\frac{\rho(s) p(s)}{c_{2} a(s)}+\frac{\rho(s) h(t, s)}{\sqrt{H(t, s)}}-\rho^{\prime}(s)\right]^{2} H(t, s) \frac{c_{2} a(s)}{k \rho(s)}\right\} \mathrm{d} s=+\infty,
\end{aligned}
$$

then Equation (1) is oscillatory.

Proof. Suppose that $x(t)$ is a nonoscillatory solution of Equation (1). We may assume without loss of generality that $x(t) \neq 0$ with $t \geq t_{0}$. we consider the function

$$
W(t)=\frac{a(t) \psi(x(t)) x^{\prime}(t)}{f(x(t))}, \quad t \geq t_{0} .
$$

From Equation (1), we get

$$
\begin{aligned}
W^{\prime}(t) & =-q(t)-\frac{p(t) x^{\prime}(t)}{f(x(t))}-a(t) \psi(x(t)) f^{\prime}(x(t)) \frac{x^{\prime 2}(t)}{f^{2}(x(t))} \\
& \leq-q(t)-\frac{1}{\psi(x(t))}\left[\frac{k}{a(t)} W^{2}(t)+\frac{p(t)}{a(t)} W(t)\right]
\end{aligned}
$$


So when $t \geq s \geq t_{0}$, we have

$$
\begin{aligned}
& =-q(t)+\frac{1}{\psi(x(t))} \frac{p^{2}(t)}{4 k a(t)}-\frac{1}{\psi(x(t))}\left[\sqrt{\frac{k}{a(t)}} W(t)+\frac{p(t)}{2 \sqrt{k a(t)}}\right]^{2} \\
& \leq-q(t)+\frac{p^{2}(t)}{4 k c_{1} a(t)}-\frac{1}{c_{2}}\left[\sqrt{\frac{k}{a(t)}} W(t)+\frac{p(t)}{2 \sqrt{k a(t)}}\right]^{2} \\
& =-q(t)-\frac{\left(c_{1}-c_{2}\right) p^{2}(t)}{4 k c_{1} c_{2} a(t)}-\frac{1}{c_{2}}\left[\frac{k}{a(t)} W^{2}(t)+\frac{p(t)}{a(t)} W(t)\right] .
\end{aligned}
$$

$$
\begin{aligned}
\int_{t_{0}}^{t} H(t, s) \rho(s) W^{\prime}(s) \mathrm{d} s \leq & -\int_{t_{0}}^{t} H(t, s) \rho(s)\left[q(s)+\frac{\left(c_{1}-c_{2}\right) p^{2}(s)}{4 k c_{1} c_{2} a(s)}\right] \mathrm{d} s \\
& -\frac{1}{c_{2}} \int_{t_{0}}^{t} H(t, s) \rho(s)\left[\frac{k}{a(t)} W^{2}(s)+\frac{p(s)}{a(s)} W(s)\right] \mathrm{d} s .
\end{aligned}
$$

By the division integral formula and applying Equation (4), we have

$$
\int_{t_{0}}^{t} H(t, s) \rho(s) W^{\prime}(s) \mathrm{d} s=-H\left(t, t_{0}\right) \rho\left(t_{0}\right) W\left(t_{0}\right)+\int_{t_{0}}^{t}\left[\frac{\rho(s) h(t, s)}{\sqrt{H(t, s)}}-\rho^{\prime}(s)\right] H(t, s) W(s) \mathrm{d} s
$$

So when $t \geq s \geq t_{0}$, it follows

$$
\begin{aligned}
& \int_{t_{0}}^{t} H(t, s) \rho(s)\left[q(s)+\frac{\left(c_{1}-c_{2}\right) p^{2}(s)}{4 k c_{1} c_{2} a(s)}\right] \mathrm{d} s \\
& \leq H\left(t, t_{0}\right) \rho\left(t_{0}\right) W\left(t_{0}\right)-\frac{k}{c_{2}} \int_{t_{0}}^{t} \frac{H(t, s) \rho(s) W^{2}(s)}{a(s)} \mathrm{d} s \\
& \quad-\int_{t_{0}}^{t}\left[\frac{\rho(s) p(s)}{c_{2} a(s)}+\frac{\rho(s) h(t, s)}{\sqrt{H(t, s)}}-\rho^{\prime}(s)\right] H(t, s) W(s) \mathrm{d} s,
\end{aligned}
$$

By (9), when $t \geq s \geq t_{0}$, we get

$$
\begin{aligned}
& \int_{t_{0}}^{t}\left\{H(t, s) \rho(s)\left[q(s)+\frac{\left(c_{1}-c_{2}\right) p^{2}(s)}{4 k c_{1} c_{2} a(s)}\right]\right. \\
& \left.-\frac{1}{4}\left[\frac{\rho(s) p(s)}{c_{2} a(s)}+\frac{\rho(s) h(t, s)}{\sqrt{H(t, s)}}-\rho^{\prime}(s)\right]^{2} H(t, s) \frac{c_{2} a(s)}{k \rho(s)}\right\} \mathrm{d} s \\
& \leq H\left(t, t_{0}\right) \rho\left(t_{0}\right) W\left(t_{0}\right)-\int_{t_{0}}^{t}\left\{\sqrt{H(t, s)} \sqrt{\frac{k \rho(s)}{c_{2} a(s)}} W(s)\right. \\
& \left.+\frac{1}{2}\left[\frac{\rho(s) p(s)}{c_{2} a(s)}+\frac{\rho(s) h(t, s)}{\sqrt{H(t, s)}}-\rho^{\prime}(s)\right] \sqrt{H(t, s)} \sqrt{\frac{c_{2} a(s)}{k \rho(s)}}\right\}^{2} \mathrm{~d} s \\
& \leq H\left(t, t_{0}\right) \rho\left(t_{0}\right) W\left(t_{0}\right) .
\end{aligned}
$$

The two sides of (10) are divided by $H\left(t, t_{0}\right)$, and we calculate the limit of the two sides of (10) when $t \rightarrow+\infty$. So we have a contradiction to the condition (5). This completes the proof.

Corollary 1. In Theorem 1, if the condition (5) is replaced by the following conditions:

1) $\limsup _{t \rightarrow+\infty} \frac{1}{H\left(t, t_{0}\right)} \int_{t_{0}}^{t} H(t, s) \rho(s)\left[q(s)+\frac{\left(c_{1}-c_{2}\right) p^{2}(s)}{4 k c_{1} c_{2} a(s)}\right] \mathrm{d} s=+\infty$, 
2)

$$
\lim _{t \rightarrow+\infty} \frac{1}{H\left(t, t_{0}\right)} \int_{t_{0}}^{t}\left[\frac{\rho(s) p(s)}{c_{2} a(s)}+\frac{\rho(s) h(t, s)}{\sqrt{H(t, s)}}-\rho^{\prime}(s)\right]^{2} H(t, s) \frac{c_{2} a(s)}{k \rho(s)} \mathrm{d} s<+\infty,
$$

then Equation (1) is oscillatory.

Remark 1. In Theorem 1, if we select different functions $\rho(t)$ and $H(t, s)$ the different oscillation criteria of Equation (1) can be obtained. For example, you can select $H(t, s)=(t-s)^{\alpha}$ or $H(t, s)=\left(\ln \frac{t+1}{s+1}\right)^{m}$.

If the condition (5) is not satisfied, we can apply the following guidelines for determining oscillation of Equation (1).

Theorem 2. Assume that $\left(A_{1}\right)-\left(A_{3}\right)$ hold, and $0<c_{1} \leq \psi(x) \leq c_{2}, f^{\prime}(x) \geq k>0, x \neq 0 . H(t, s)$ belongs to the class of functions $P$ and (4) holds. Besides,

$$
0<\inf _{s \geq t_{0}}\left\{\liminf _{t \rightarrow \infty} \frac{H(t, s)}{H\left(t, t_{0}\right)}\right\} \leq \infty .
$$

If there is a continuously differentiable function $\rho(t):\left[t_{0},+\infty\right) \rightarrow(0,+\infty)$ to make

$$
\limsup _{t \rightarrow+\infty} \frac{1}{H\left(t, t_{0}\right)} \int_{t_{0}}^{t}\left[\frac{\rho(s) p(s)}{c_{2} a(s)}+\frac{\rho(s) h(t, s)}{\sqrt{H(t, s)}}-\rho^{\prime}(s)\right]^{2} H(t, s) \frac{c_{2} a(s)}{k \rho(s)} \mathrm{d} s<+\infty,
$$

and continuously function $\varphi(t):\left[t_{0},+\infty\right) \rightarrow \mathrm{R}$ to make

$$
\begin{aligned}
& \liminf _{t \rightarrow+\infty} \frac{1}{H(t, s)} \int_{s}^{t}\left\{H(t, \tau) \rho(\tau)\left[q(\tau)+\frac{\left(c_{1}-c_{2}\right) p^{2}(\tau)}{4 k c_{1} c_{2} a(\tau)}\right]\right. \\
& \left.-\frac{1}{4}\left[\frac{\rho(\tau) p(\tau)}{c_{2} a(\tau)}+\frac{\rho(\tau) h(t, \tau)}{\sqrt{H(t, \tau)}}-\rho^{\prime}(\tau)\right]^{2} H(t, \tau) \frac{c_{2} a(\tau)}{k \rho(\tau)}\right\} \mathrm{d} \tau \geq \varphi(s)
\end{aligned}
$$

hold when $t \geq s \geq t_{0}$. Besides,

$$
\lim _{t \rightarrow+\infty} \frac{1}{H\left(t, t_{0}\right)} \int_{t_{0}}^{t} H(t, s) \frac{\varphi_{+}^{2}(s)}{\rho(s) a(s)} \mathrm{d} s=+\infty,
$$

where $\varphi_{+}(t)=\max \{\varphi(t), 0\}$. Then Equation (1) is oscillatory.

Proof. Suppose that $x(t)$ is a nonoscillatory solutions of (1). And when $t \geq t_{0}, x(t) \neq 0$. Define

$$
W(t)=\frac{a(t) \psi(x(t)) x^{\prime}(t)}{f(x(t))}, \quad t \geq t_{0} .
$$

We can get (10) as the proof of Theorem 1, i.e.

$$
\begin{aligned}
& \int_{s}^{t}\left\{H(t, \tau) \rho(\tau)\left[q(\tau)+\frac{\left(c_{1}-c_{2}\right) p^{2}(\tau)}{4 k c_{1} c_{2} a(\tau)}\right]\right. \\
& \left.-\frac{1}{4}\left[\frac{\rho(\tau) p(\tau)}{c_{2} a(\tau)}+\frac{\rho(\tau) h(t, \tau)}{\sqrt{H(t, \tau)}}-\rho^{\prime}(\tau)\right]^{2} H(t, \tau) \frac{c_{2} a(\tau)}{k \rho(\tau)}\right\} \mathrm{d} \tau \\
& \leq H(t, s) \rho(s) W(s), \quad t \geq s \geq t_{0} .
\end{aligned}
$$

The two sides of the above result are divided by $H\left(t, t_{0}\right)$, then we calculate the limit of the two sides when $t \rightarrow+\infty$. By (14), we get $\varphi(s) \leq \rho(s) W(s), s \geq t_{0}$. So

$$
\varphi_{+}^{2}(s) \leq \rho^{2}(s) W^{2}(s) .
$$


Define

$$
\begin{aligned}
& u(t)=\frac{1}{H\left(t, t_{0}\right)} \int_{t_{0}}^{t}\left[\frac{\rho(s) p(s)}{c_{2} a(s)}+\frac{\rho(s) h(t, s)}{\sqrt{H(t, s)}}-\rho^{\prime}(s)\right] H(t, s) W(s) \mathrm{d} s, t>t_{0} ; \\
& v(t)=\frac{1}{H\left(t, t_{0}\right)} \frac{k}{c_{2}} \int_{t_{0}}^{t} \frac{H(t, s) \rho(s) W^{2}(s)}{a(s)} \mathrm{d} s, t>t_{0} .
\end{aligned}
$$

By (9), we have

$$
u(t)+v(t) \leq \rho\left(t_{0}\right) W\left(t_{0}\right)-\frac{1}{H\left(t, t_{0}\right)} \int_{t_{0}}^{t} H(t, s) \rho(s)\left[q(s)+\frac{\left(c_{1}-c_{2}\right) p^{2}(s)}{4 k c_{1} c_{2} a(s)}\right] \mathrm{d} s,
$$

and by (14), we get

$$
\liminf _{t \rightarrow+\infty} \frac{1}{H(t, s)} \int_{s}^{t} H(t, \tau) \rho(\tau)\left[q(\tau)+\frac{\left(c_{1}-c_{2}\right) p^{2}(\tau)}{4 k c_{1} c_{2} a(\tau)}\right] \mathrm{d} \tau \geq \varphi(s), s \geq t_{0}
$$

and

$$
\begin{aligned}
& \limsup _{t \rightarrow+\infty} \frac{1}{H\left(t, t_{0}\right)} \int_{t_{0}}^{t} H(t, s) \rho(s)\left[q(s)+\frac{\left(c_{1}-c_{2}\right) p^{2}(s)}{4 k c_{1} c_{2} a(s)}\right] \mathrm{d} s \\
& -\liminf _{t \rightarrow+\infty} \frac{1}{4} \frac{1}{H\left(t, t_{0}\right)} \int_{t_{0}}^{t}\left[\frac{\rho(s) p(s)}{c_{2} a(s)}+\frac{\rho(s) h(t, s)}{\sqrt{H(t, s)}}-\rho^{\prime}(s)\right]^{2} H(t, s) \frac{c_{2} a(s)}{k \rho(s)} \mathrm{d} s \geq \varphi\left(t_{0}\right) .
\end{aligned}
$$

By (13) and (22) there is a sequence

$$
\left\{t_{n}\right\}_{1}^{\infty}, t_{n}>t_{0}, n=1,2,3, \cdots, \lim _{n \rightarrow+\infty} t_{n}=+\infty,
$$

such that

$$
\lim _{n \rightarrow+\infty} \frac{1}{4} \frac{1}{H\left(t_{n}, t_{0}\right)} \int_{t_{0}}^{t_{n}}\left[\frac{\rho(s) p(s)}{c_{2} a(s)}+\frac{\rho(s) h\left(t_{n}, s\right)}{\sqrt{H\left(t_{n}, s\right)}}-\rho^{\prime}(s)\right]^{2} H\left(t_{n}, s\right) \frac{c_{2} a(s)}{k \rho(s)} \mathrm{d} s<+\infty .
$$

When $t \rightarrow+\infty$ we calculate the supper limit of (20) and apply (21), it follows

$$
\limsup _{t \rightarrow+\infty}\{u(t)+v(t)\} \leq \rho\left(t_{0}\right) W\left(t_{0}\right)-\varphi\left(t_{0}\right)=\beta .
$$

So for sufficiently large $n$, there is

$$
u\left(t_{n}\right)+v\left(t_{n}\right)<\beta
$$

Because

$$
v(t)=\int_{t_{0}}^{t} \frac{H(t, s)}{H\left(t, t_{0}\right)} \rho(s) \frac{k W^{2}(s)}{c_{2} a(s)} \mathrm{d} s>0, t>t_{0}
$$

is increasing, we get $\lim v(t)=C$ Where $C=+\infty$ or is a positive constant. Assume that $C=+\infty$, then $\lim _{n \rightarrow+\infty} v\left(t_{n}\right)=+\infty$ and $\overrightarrow{b y}^{+\infty}(26)$, we have

$$
\lim _{n \rightarrow+\infty} u\left(t_{n}\right)=-\infty
$$

From (26) and (28), $\frac{u\left(t_{n}\right)}{v\left(t_{n}\right)}+1<\varepsilon$, where $0<\varepsilon<1$ is a constant. That is for sufficiently large $t_{n}$

$$
\frac{u\left(t_{n}\right)}{v\left(t_{n}\right)}<\varepsilon-1<0
$$


On the other hand, by the Schwarz inequality, we get

$$
\begin{aligned}
0 \leq & \frac{1}{H^{2}\left(t_{n}, t_{0}\right)}\left(\int_{t_{0}}^{t_{n}}\left[\frac{\rho(s) p(s)}{c_{2} a(s)}+\frac{\rho(s) h\left(t_{n}, s\right)}{\sqrt{H\left(t_{n}, s\right)}}-\rho^{\prime}(s)\right] H\left(t_{n}, s\right) W(s) \mathrm{d} s\right)^{2} \\
\leq & \left(\frac{1}{H\left(t_{n}, t_{0}\right)} \int_{t_{0}}^{t_{n}}\left[\frac{\rho(s) p(s)}{c_{2} a(s)}+\frac{\rho(s) h\left(t_{n}, s\right)}{\sqrt{H\left(t_{n}, s\right)}}-\rho^{\prime}(s)\right]^{2} H\left(t_{n}, s\right) \frac{c_{2} a(s)}{k \rho(s)} \mathrm{d} s\right) \\
& \cdot\left(\frac{1}{H\left(t_{n}, t_{0}\right)} \int_{t_{0}}^{t_{n}} H\left(t_{n}, s\right) \frac{k \rho(s)}{c_{2} a(s)} W^{2}(s) \mathrm{d} s\right) .
\end{aligned}
$$

So

$$
0 \leq \frac{u^{2}\left(t_{n}\right)}{v\left(t_{n}\right)} \leq \frac{1}{H\left(t_{n}, t_{0}\right)} \int_{t_{0}}^{t_{n}}\left[\frac{\rho(s) p(s)}{c_{2} a(s)}+\frac{\rho(s) h\left(t_{n}, s\right)}{\sqrt{H\left(t_{n}, s\right)}}-\rho^{\prime}(s)\right]^{2} H\left(t_{n}, s\right) \frac{c_{2} a(s)}{k \rho(s)} \mathrm{d} s .
$$

From (24), we have

$$
0 \leq \lim _{n \rightarrow+\infty} \frac{u^{2}\left(t_{n}\right)}{v\left(t_{n}\right)}<+\infty .
$$

There is an contradiction with (28) and (29). If $\lim _{t \rightarrow+\infty} v(t)=C<+\infty$ with (18) we get

$$
\lim _{t \rightarrow+\infty} \frac{k}{c_{2}} \frac{1}{H\left(t, t_{0}\right)} \int_{t_{0}}^{t} H(t, s) \frac{\varphi_{+}^{2}(s)}{\rho(s) a(s)} \mathrm{d} s \leq \lim _{t \rightarrow+\infty} \frac{k}{c_{2}} \frac{1}{H\left(t, t_{0}\right)} \int_{t_{0}}^{t} H(t, s) \rho(s) \frac{W^{2}(s)}{a(s)} \mathrm{d} s=C<+\infty,
$$

we obtain a contradiction to (15). This completes the proof.

Remark 2. The theorems of this paper improve or extend the results in [1]-[12]. For Equation (1), Theorem 1 and 2 are new.

Finally, we give two examples.

Example 1. Consider the second-order differential equation with damping

$$
\left[\left(1+\sin ^{2} x(t)\right) x^{\prime}(t)\right]^{\prime}-\frac{1}{t} x^{\prime}(t)+\frac{1}{t^{\frac{3}{2}}}\left[x^{3}(t)+x(t)\right]=0, t \geq t_{0}:=1,
$$

where $a(t)=1, \psi(u)=1+\sin ^{2} u, q(t)=t^{-\frac{3}{2}}, p(t)=-t^{-1}, f(u)=u^{3}+u$.

Now let $H(t, s)=(t-s)^{2}, h(t, s)=2, \rho(t)=t$, It is easy to verify that Equation (34) satisfies all the conditions of Theorem 1, so by Theorem 1, Equation (34) is oscillatory.

Example 2. Consider the second-order differential equation with damping

$$
\left[\frac{1}{t^{2}} x^{\prime}(t)\right]^{\prime}+\frac{1}{t^{4}} x^{\prime}(t)+\frac{1}{t^{3}} x(t)=0, t \geq t_{0}:=2
$$

Here $a(t)=\frac{1}{t^{2}}, \psi(u)=1, p(t)=\frac{1}{t^{4}}, q(t)=\frac{1}{t^{3}}, f(u)=u$.

Now let $H(t, s)=(t-s)^{2}, h(t, s)=2, \rho(t)=t, \varphi(t)=\frac{1}{2 t}$, so all the conditions of Theorem 2 are satisfied. By Theorem 2, Equation (35) is oscillatory on $\left[t_{0}, \infty\right)$. But the other known results cannot be applied in Equation (35).

\section{Conclusions and Outlook}

In this paper, the two well-known results of Philos on the second order linear differential equation are extended 
to the second order nonlinear differential equations with damping term. As we all know, the motions under ideal conditions and vacuum are rare, but the motions with damping and disturbances are widespread. The discussion on the oscillation of the differential equation with damping term in our paper is of more practical significance. Moreover, the previous study on oscillation of the equation always assumed that $p(t)>0, q(t)>0$, but the sign of $p(t)$ and $q(t)$ in our paper may change. Therefore, in this paper we extend and improve some of the results that are known in the previous study.

It is a deficiency of this paper that there is no discussion on delay. So in the follow-up study we will discuss the oscillation of the second order delay differential equations with damping, second order neutral delay differential equations and higher order delay differential equations with damping.

\section{Acknowledgements}

We thank the Editor and the referee for their comments. Research of Q. Zhang is funded by the Natural Science Foundation of Shandong Province of China grant ZR2013AM003. This support is greatly appreciated.

\section{References}

[1] Zhang, Q. and Yan, J. (2004) The Oscillation of the Second Order Nonlinear Differential Equations with Damping. Journal of Systems Science and Mathematical Science, 24, 296-302. (In Chinese)

[2] Zhang, Q. and Yan, J. (2007) Oscillatory Behavior of Second Order Nonlinear Differential Equation with Damping. Journal of Mathematics, 27, 455-460. (In Chinese)

[3] Zhang, Q. and Yan, J. (2008) Oscillatory Behavior of the Second Order Nonlinear Delay Differential Equation. Pure and Applied Mathematics, 24, 646-653. (In Chinese)

[4] Yan, J. (1986) Oscillatory Theorems for Second Order Linear Differential Equations with Damping. Proceedings of the American Mathematical Society, 98, 276-282. http://dx.doi.org/10.1090/S0002-9939-1986-0854033-4

[5] Cecchi, M. and Marini, M. (1992) Oscillatory and Nonoscillatory Behavior of a Second Order Functional Differential Equation. Rocky Mountain Journal of Mathematics, 22, 1259-1276. http://dx.doi.org/10.1216/rmjm/1181072653

[6] Rogovchenko, Yu.V. (2000) On Oscillation of a Second Order Nonlinear Delay Differential Equation. Funkcialaj Ekvacioj, 43, 1-29.

[7] Yeh, C.C. (1989) Oscillation Theorems for Second Order Nonlinear Perturbed Differentional Equations. Journal of Mathematical Analysis and Applications, 138, 157-165. http://dx.doi.org/10.1016/0022-247X(89)90326-0

[8] Philos, Ch.G. (1989) Oscillation Theorems for Linear Differential Equations of Second Order. Archiv der Mathematik, 53, 482-492. http://dx.doi.org/10.1007/BF01324723

[9] Zhang, Q., Liu, S. and Gao, L. (2011) Oscillation Criteria for Even-Order Half-Linear Functional Differentional Equations with Damping. Applied Mathematics and Letters, 24, 1709-1715. http://dx.doi.org/10.1016/j.aml.2011.04.025

[10] Gao, L., Zhang, Q. and Liu, S. (2015) Oscillatory Behavior of Second-Order Half-Linear Neutral Differential Equations with Damping Term. Advanced Studies in Contemporary Mathematics, 25, 463-471.

[11] Zhang, Q., Gao, L. and Wang, S. (2012) Oscillatory and Asymptotic Behavior of a Second-Order Nonlinear Functional Differentional Equations. Communications in Theoretical Physics, 57, 914-922. http://dx.doi.org/10.1088/0253-6102/57/6/02

[12] Song, X. and Zhang, Q. (2014) Bounded Oscillation for Second-Order Nonlinear Delay Differential Equations. Journal of Applied Mathematics \& Informatics, 32, 447-454. http://dx.doi.org/10.14317/jami.2014.447 